RAE-IC, Revista de la Asociación Española de Investigación de la Comunicación vol. 8, núm. 16 (2021), 51-71 ISSN 2341-2690

Recibido el 10 de agosto de 2021 DOI: https://doi.org/10.24137/raeic.8.16.4 Aceptado el 21 de septiembre de 2021

\title{
La brecha de género en los Oscar: análisis de los premios concedidos a mujeres entre el 2000 y el 2020
}

Gender gap in the Oscar's: analysis of prizes awarded to women between 2000 and 2020

Rodríguez-Martelo, Talía

Universidade de Vigo (UVIGO)

talia.rodriguez@uvigo.es

Maroto González, Isaac Universidade de Santiago de Compostela (USC) isaac.maroto.gonzalez@usc.es

Fontenla-Pedreira, Julia Universidade de Vigo (UVIGO) julia.fontenla.pedreira@uvigo.es

Forma de citar este artículo:

Rodríguez-Martelo, T. Maroto González, I. y Fontenla-Pedreira, J. (2021). La brecha de género en los Oscar: análisis de los premios concedidos a mujeres entre el 2000 y el 2020. RAEIC, Revista de la Asociación Española de Investigación de la Comunicación, 8(16), 51-71.

https://doi.org/10.24137/raeic.8.16.4 


\section{Resumen:}

El techo de cristal es un concepto que define la dificultad que encuentran las mujeres para acceder a ciertas posiciones en el sector laboral. De forma habitual se comprueba cómo cuanto más se asciende en la escala de puestos directivos menor es la presencia de mujeres debido a que su carrera se trunca durante su vida laboral antes de tener la oportunidad de avanzar hacia puestos elevados. La industria cinematográfica es un sector que no parece escapar a esta lógica de desigualdad y, por eso, el presente estudio se ocupa de analizar la presencia de las mujeres en un certamen tan relevante como los premios Oscar concedidos por la Academia de las Artes y las Ciencias Cinematográficas. Se ha priorizado la elección de este ejemplo sobre otros debido a la relevancia internacional del certamen como referencia de la industria del cine a nivel mundial, además de la disponibilidad de información que ofrece la entidad. La metodología empleada está basada en un enfoque cuantitativo y cualitativo de revisión de fuentes documentales y análisis de datos obtenidos de bases de datos de acceso abierto. Los resultados procesados y codificados muestran como las mujeres se encuentran infrarrepresentadas en la concesión de premios en el ejercicio de la profesión audiovisual a pesar de que las cifran tienden a aumentar conforme pasan los años.

Palabras clave: cine, Oscars, igualdad, MeToo, mujer, industria audiovisual.

\section{Abstract:}

Glass ceiling is a concept which commonly defines how difficult is for the women to access to high positions on the labour market. Usually, it can be seen how high is the step on social scale the less are the presence of women due to their career results stacked before even appears the chance to get a high-level position. Cinema industry is a field which seems unable to escape from this inequality logic, so this article aims to analyse the representation of women in such a relevant event as Oscar's Awards by Academy of Motion Picture Arts and Science. Has been prioritized the election of this festival among others given its international importance, furthermore the open access data base offered by the institution. Methodology is based on a quantitative and 
qualitative approach developed through a review of documentary sources and analyse of information obtained from open data bases. Results processed and codified shows how women are misrepresented on the awards and social recognition for the audiovisual careers even the numbers tends to increase year after year.

Keywords: cinema, equality, Oscar's, women, MeToo, audiovisual industry

\section{INTRODUCCIÓN}

La representación de la mujer en el medio audiovisual es un tema recurrente en los estudios de género. Como se produce la construcción de personajes femeninos en películas, en series o en la televisión tiene una relación directa con el imaginario social colectivo y con la ideología dominante. Las mujeres profesionales que ocupan los cargos de dirección y los roles creativos detrás de las cámaras apenas suponen el 20\% (Lauzen, 2018). A pesar de que hoy en día la sociedad se encuentra en medio de la corriente transformadora de la cuarta ola feminista, en la mayoría de sectores laborales las mujeres siguen enfrentando graves desigualdades y el techo de cristal les impide ascender a puestos directivos o relacionados con la toma de decisiones. En este sentido, en la industria cinematográfica la desigualdad se manifiesta desde una perspectiva múltiple: por una parte, con la mujer representada en la pantalla y, por otra, en la ausencia de mujeres en determinados puestos laborales. El presente estudio plantea como esta baja participación de las mujeres detrás de la cámara se manifiesta en función de la visibilidad del colectivo femenino en los festivales de cine y los certámenes de entregas de premios y eventos de la industria que tienen como objetivo el reconocimiento en el ejercicio de una profesión determinada.

\section{MARCO TEÓRICO}

\subsection{EL ACTIVISMO FEMINISTA Y LA REVOLUCIÓN \#METOO}

En el siglo XXI han tenido lugar numerosos eventos relacionados con el activismo y la defensa de la igualdad en derechos de diferentes colectivos. La denominada sociedad 
post 11-S experimentó cambios sustanciales en la forma de enfrentar las problemáticas colectivas, la política, la economía y la comunicación (Bazo Varela, 2003) en un nuevo contexto digital dominado por las redes sociales. Este nuevo paradigma de comunicación ha servido como vehículo transformador en las dinámicas relacionadas con las demandas de derechos civiles debido a su poder como herramienta única para la organización de movimientos sociales y la difusión de información.

Durante la última década el activismo feminista ha sido de enorme relevancia materializándose como lucha colectiva a través de diferentes eventos y en función de diversos enfoques. A pesar de que el feminismo tiene una larga trayectoria en su definición, estructura y divulgación, la tercera ola que se inicia en los años noventa se extiende hasta la segunda década del nuevo siglo y los avances conseguidos se van traduciendo en políticas concretas y nuevas legislaciones, así como con la proliferación de los estudios de género (Saavedra, 2019). En este sentido, es innegable el papel desarrollado por los medios sociales y el uso popular y cotidiano que adquieren muchos conceptos relacionados con la defensa de la igualdad y la lucha feminista (AlonsoGonzález, 2021).

La cuarta ola del feminismo en la que estamos inmersos hoy en día se inicia a partir del movimiento \#MeToo. En el año 2017 el productor de cine Harvey Weinstein es acusado de abuso sexual por varias actrices. Según avanzan los días desde la primera acusación, la lista de mujeres que denuncian a Weinstein se va incrementando con nombres de artistas de fama mundial como Angelina Jolie o Gwyneth Paltrow. El 15 de octubre de ese mismo año la actriz Alyssa Milano hace un llamamiento a través de Twitter sugiriendo que todas las mujeres víctimas de abuso o acoso sexual utilicen el hashtag \#MeToo. Las redes se inundan con testimonios de mujeres y el 25 de octubre la revolución MeToo se extiende hasta el parlamento europeo. El año siguiente el movimiento tiene un gran protagonismo en los Globos de Oro y en los premios Oscars. La huelga feminista del 8 de marzo de 2018 adquiere una dimensión y repercusión internacional. Al hashtag \#MeToo se le añaden otros que se convierten también en tendencia cómo \#Time’sUp, \#Yositecreo, \#Cuéntalo o \#BelieveSurvivors. El movimiento 
continúa presente en medios de comunicación y de forma transnacional se va relacionando con diferentes eventos y colectivos activistas como la defensa del aborto en Argentina o las actrices africanas. El 26 de noviembre el grupo activista Las tesis realiza la performance "Un violador en tu camino" en Santiago de Chile, en pocos días se replica esta acción por todo el mundo en diversos países y en diferentes idiomas. Se suman nuevas denuncias de personajes reconocidos y se prolonga el desarrollo del movimiento hasta el inicio del juicio de Weinstein en 2020 (El País, 2020).

\subsection{LA PARTICIPACIÓN Y REPRESENTACIÓN DE LA MUJER EN LA INDUSTRIA} AUDIOVISUAL

La representación de la mujer en el sector cinematográfico y las contradicciones derivadas de cómo se construye la imagen femenina en el relato audiovisual es un tema recurrente en los estudios de género. Aunque este abordaje concreto no se relaciona de forma directa con el objeto de estudio planteado en esta investigación, si es relevante y necesario hacer un recorrido exploratorio por algunos trabajos destacados que arrojan luz sobre conceptos y problemáticas que ilustran de forma clara y precisa el tema central tratado. El tratamiento que recibe el género femenino delante de la cámara tiene un reflejo en la infrarrepresentación detrás de la misma.

En los estereotipos audiovisuales las mujeres destacan por la importancia que se otorga a su físico (Guarinos, 2008) y por los parámetros bajo los cuáles se desarrollan sus personajes. Tanto si hablamos de arquetipos tan dispares como "la madre", "la mediadora", "el oráculo" o la "heroína solitaria", entre otros, cuando se trata de protagonistas femeninas se asocian con valores como la humanidad o la sensibilidad (Checa y Adell, 2019).

Para los personajes masculinos la narrativa audiovisual se articula en función del viaje del héroe y los arquetipos a los que responden los personajes hombres tienen mayor profundidad en el desarrollo de los relatos. Estudios como el desarrollado mediante el Test de Bechdel (Kelley, 2014) o investigaciones similares como la de Zhao (2019) que 
aplica el machine learning en el análisis de diálogos de películas vienen a ilustrar cómo la brecha de género continúa patente en el desarrollo narrativo audiovisual.

Históricamente la mujer está cosificada, sobre ella se proyecta el deseo masculino y es un ser pasivo (Castejón Leorza, 2004). Esta dialéctica narrativa se construye a partir de lo femenino/objeto/pasivo mientras que lo masculino está representado por el hombre/cultura/activo (Yates, 2017). A pesar de que se observa un cambio en los relatos desde hace años y, de forma más exhaustiva, desde el movimiento \#MeToo que parece haber acelerado el posicionamiento de la industria cinematográfica y del sector audiovisual en general, la realidad aún es poco prometedora y plagada de estereotipos machistas.

Para trabajar sobre parámetros de igualdad, la representación de la mujer debe ser proporcional en términos narrativos y de fuerza de trabajo y en posiciones de toma de decisiones dentro y fuera de la pantalla (Cabrera et al., 2021). Sin embargo, no existe una correspondencia entre el número de mujeres que trabajan en la industria y las mujeres que ostentan puestos de responsabilidad o claves en el control creativo de los proyectos cinematográficos y audiovisuales en general.

Tal y como exponen los resultados del estudio de Annenberg Foundation (2020), el perfil femenino representado en la pantalla es una mujer joven - menos de 45 años-, blanca y en roles secundarios o coprotagonistas. Este estudio que analiza 1300 películas entre 2007 y 2019 ha determinado que existe ausencia de protagonistas femeninas únicas (solo 43 en total), de grupos raciales distintos al caucásico (tan solo 17 actrices) y mayores de 45 años (únicamente en 3 ocasiones).

Según este mismo estudio, en el año 2019, último del que ofrece datos, tan solo el 10’7\% de la dirección cinematográfica estaba ejecutada por mujeres, un 19`4\% eran guionistas y un 24'3\% han sido productoras (Annenberg, 2020). Estos datos no son más prometedores en otros contextos ya que tal y como recogen Fontaine y Simone (2020), en Europa, solo un 22\% de los trabajos de dirección entre 2015 y 2018 están asumidos por mujeres y solo un $25 \%$ en el caso de las guionistas. 
De forma habitual las mujeres han sido relegadas en la historia del cine, la ausencia de nombres femeninos se extiende por la mayoría de manuales de historia de la industria poniendo en evidencia el sistema patriarcal (Berrueta, 2019). La participación femenina en las diferentes áreas de la producción cinematográfica más allá del rol de actrices viene a ser difundida por los estudios de género que ofrecen otra perspectiva en las que las mujeres también han sido clave en el desarrollo de la cinematografía mundial como, por ejemplo, la serie documental Women Make Film (Cousins, 2018). Estrenada en 2018, esta exhaustiva investigación viene a corroborar el trabajo realizado por las mujeres en la historia del cine como directoras, guionistas, directoras de fotografía, realizadoras o productoras y su capacidad transformadora en la innovación cinematográfica.

\subsection{LOS OSCAR: UN CERTÁMEN DE CINE SIN PERSPECTIVA DE GÉNERO}

Los festivales de cine, entregas de premios y eventos de la industria audiovisual constituyen un escaparate del cine del momento que sirve como elemento promocional de las películas concretas de cada edición, así como de la cultura cinematográfica en general. Cuando se habla de la perspectiva de género, los festivales de cine resultan de vital importancia para dar visibilidad a las mujeres profesionales. Es habitual que el techo de cristal que sufren las mujeres en la mayoría de las profesiones también esté presente en el sector audiovisual. Por ello, cuando se pone el foco de atención en determinados puestos laborales y cuanto más cualificados y con más responsabilidad son éstos, menor cantidad de mujeres se encuentran al frente. Sin embargo, la profesión concentra mayor índice femenino en puestos de menor cualificación (HernándezCarrillo y Ruiz del Olmo, 2018; Roquero, 2012).

Algunos de los eventos más famosos e influyentes del mundo relacionados con la industria cinematográfica son los premios Oscar celebrados anualmente en Los Ángeles (Estados Unidos), la Berlinale en Berlín (Alemania), el festival de cine de Cannes (Francia), los premios BAFTA (Inglaterra) o el Festival Internacional de Cine de San Sebastián (España), entre otros. También existen certámenes que no solo premian cine y son de gran relevancia internacional como, por ejemplo, los Globos de Oro también celebrados en Estados Unidos. 
De todos los eventos relacionados con la industria audiovisual, los premios concedidos por la Academia de las Artes y Ciencias de la Cinematografía, comúnmente denominados los Oscar de Hollywood, es quizás, la cita más famosa a nivel internacional debido a la amplia circulación de sus películas, su puesta en escena y el star system que lo avala. Su alfombra roja es noticia en los informativos de todo el mundo cada vez que se celebra el evento.

Este certamen se ha desarrollado de forma ininterrumpida desde 1929 y en él se premian diferentes categorías relacionadas con todas las funciones que intervienen en la realización de una película. De todas las áreas en las que se conceden galardones, las de mayor popularidad suelen ser la Mejor Película, Mejor Dirección y Mejor Actor y Actriz en papeles protagonistas. Esta categoría y la de Mejor Actor y Actriz en papeles secundarios son las únicas que presentan una distribución basada en el género. En el resto de las categorías no existe esta diferenciación. Cada año se entregan alrededor de 27 galardones dependiendo de la edición y, según ha ido avanzando la historia del cine y al compás de los cambios tecnológicos, se han ido agregando categorías más acordes a los nuevos modelos de producción de películas como, por ejemplo, el premio destinado a premiar los mejores efectos especiales.

En el año 2009 el certamen de los premios Oscars de Hollywood era noticia por un acontecimiento que nunca había sucedido: Katrhyn Bigelow era la primera mujer en recibir un Oscar a la mejor dirección. Su película The Hurt locker / En tierra hostil (Bigelow, 2008) también fue galardonada con el premio a Mejor película y el premio al Mejor Montaje. Nuevamente en el año 2020 una mujer se alza con la estatuilla a Mejor Dirección y Mejor Película, Chloé Zhao por la película Nomadland (Zhao, 2019). Han transcurrido once años entre ambas directoras premiadas lo cuál pone de manifiesto de forma reiterada la ausencia de nombres femeninos en los premios principales.

Dado que las entregas de premios en general y, de forma concreta, los Oscar de la Academia de Artes y Ciencias Cinematográficas representan las tendencias y novedades de la industria, su importancia en el panorama audiovisual internacional es muy elevada en tanto que suponen un termómetro de cómo se desarrolla el cine en la sociedad del 
momento, qué temas reciben interés en un momento dado o que alusiones a la actualidad se destacan tanto durante la gala como a través de las obras que se postulan.

\section{METODOLOGÍA}

Al examinar las referencias bibliográficas sobre la mujer en el entorno audiovisual se puede observar cómo es posible encontrar un gran número de estudios sobre la representación femenina en el relato audiovisual. Sin embargo, la participación de la mujer en esta industria es un tema menos analizado. La infrarrepresentación femenina en determinados puestos laborales relacionados con el sector audiovisual pone de manifiesto que es un área temática relevante para aportar una mirada más amplia a la problemática que representan los techos de cristal en la mayor parte de actividades laborales.

Este estudio trabaja bajo la premisa de que la dificultad que experimentan las mujeres para acceder a determinados puestos laborales se ve también reflejada en el reconocimiento que supone el ejercicio de una determinada profesión. En el caso del sector audiovisual o del cine, el reconocimiento a los mejores profesionales de la industria tiene su equivalencia a través de los diferentes festivales de cine de los cuáles los Oscars, que se celebran en Los Ángeles en Estados Unidos, es uno de los más importantes en el panorama internacional, además de ser el más conocido a nivel mundial.

Debido a su fama global y que el tipo de películas que se valoran en este certamen son de circulación internacional y con una elevada cuota de pantalla, se ha considerado que los Oscar eran el punto de partida idóneo para analizar la presencia de la mujer delante y detrás de la cámara y en qué medida reciben reconocimiento por su trabajo. En investigaciones como la de Hernández-Carrillo y Ruiz del Olmo (2018) se ha analizado la representación femenina bajo parámetros y premisas similares en un estudio sobre el papel que ocupan las mujeres en los diferentes ámbitos profesionales en los premios concedidos en el Festival de Málaga. 
La presente propuesta es un estudio exploratorio que parte de la premisa de que no existe una representación proporcionada de mujeres en puestos laborales de rango elevado dentro de la industria cinematográfica y esta hipótesis se comprueba contabilizando el reconocimiento en forma de premios o galardones que reciben las profesionales del audiovisual.

Para ello, se han establecido los siguientes objetivos generales y específicos de investigación:

OG1: Analizar los premios recibidos por mujeres profesionales de la industria cinematográfica en los Oscars en el período temporal comprendido entre el año 2000 y 2020.

OE1: Analizar la diferente representación en función de las categorías premiadas para valorar si existe un patrón en relación con los premios que reciben las mujeres.

OE2: Examinar el período temporal para evaluar si existe un cambio en la tendencia de representación femenina en función de los eventos y movimientos sociales acontecidos durante el período analizado.

El enfoque metodológico de este estudio se articula en función de una revisión de fuentes documentales secundarias y el tratamiento de bases de datos con información cuantitativa de la industria audiovisual. La triangulación de datos cuantitativos e información cualitativa (Gaitán y Piñuel, 1998) ha permitido examinar de forma pormenorizada el objeto de estudio. Además, la perspectiva metodológica de la triangulación aunando lo cualitativo y lo cuantitativo en la que se sistematiza la búsqueda de información aporta la capacidad de adaptar la investigación al dinamismo existente en el contexto social actual pudiendo agregar otras fuentes relevantes como redes sociales, por ejemplo, que no gozan de la precisión necesaria para trabajar por sí solas y es necesario emplear más de una estrategia metodológica (Gómez-Diago, 2010).

Dadas la limitaciones obvias que presenta la investigación en cuanto a establecer una causalidad entre la inclusión como fuerza de trabajo y la concesión de galardones en 
festivales de cine, se ha trabajado sobre esta comparativa a modo de indicativo de representación. En el análisis se han discriminado las categorías específicas que se distinguen por género como Mejor Actor/Actriz principal y Mejor Actor/Actriz en papeles secundarios debido al sesgo implícito en este dato. Este tratamiento de los datos está basado en la metodología empleada en Cuenca Suárez $(2018,2019)$ en la confección de los informes CIMA sobre la representatividad de la mujer en el sector cinematográfico del largometraje español o en Hernández-Carrillo y Ruiz del Olmo (2018).

La base del corpus de análisis está formada por los datos cuantitativos y de acceso abierto que proporcionan los Oscar sobre todos los premiados y todas las categorías mediante la Academy Awards Data Base, una base de datos de acceso abierto a todas las categorías, nominados y premiados a lo largo de la trayectoria del certamen. De forma concreta, la institución de los Oscar de Hollywood a través de la Academia de las Artes y Ciencias Cinematográficas ofrece también una base de datos sobre mujeres premiadas a lo largo de toda su historia (Academy of Motion Picture Arts and Science, 2021). A continuación, se ha establecido una tabla de codificación y se ha utilizado el software Tableau con el que se han podido analizar los datos a través del filtrado y cruce de variables. Este sistema ha permitido obtener los resultados que se presentan en el presente trabajo.

Se ha realizado una segmentación temporal de veinte años coincidente con los certámenes que han tenido lugar desde el año 2000. Esta revisión ofrece un marco amplio para juzgar cómo ha evolucionado la visibilización de la mujer como profesional de la industria cinematográfica a la luz del nuevo siglo y los acontecimientos protagonizados por colectivos activistas por la igualdad.

El análisis de resultados se ha complementado con los datos cuantitativos de los informes de Annerberg Foundation (2020), Inequality in 1,300 Popular Films: Examining Portrayals of Gender, Race/Ethnicity, LGBTQ \& Disability from 2007 to 2019, Cabrera et al. (2021), Diversity and Inclusion in the European Audiovisual Sector. Iris Plus realizado por el Observatorio Audiovisual Europeo. 


\section{RESULTADOS}

Una vez analizados los datos abiertos ofrecidos por la propia Academia de las Artes y Ciencias Cinematográficas, la codificación de los mismos en función de los objetivos marcados en la metodología se ha resuelto a partir de la confección de diferentes gráficas que exponen la evolución del papel y la representación de la mujer en la industria cinematográfica a partir del reconocimiento profesional recibido en el certamen más famoso a nivel mundial.

En primer lugar, se han explorado los datos genéricos contabilizando un total de 182 entradas que suponen el total de los premios recibidos por mujeres entre el años 2000 y el año 2020.

Tabla 1. Premios Oscar recibidos por mujeres. Período 2000-2020. Elaboración propia.

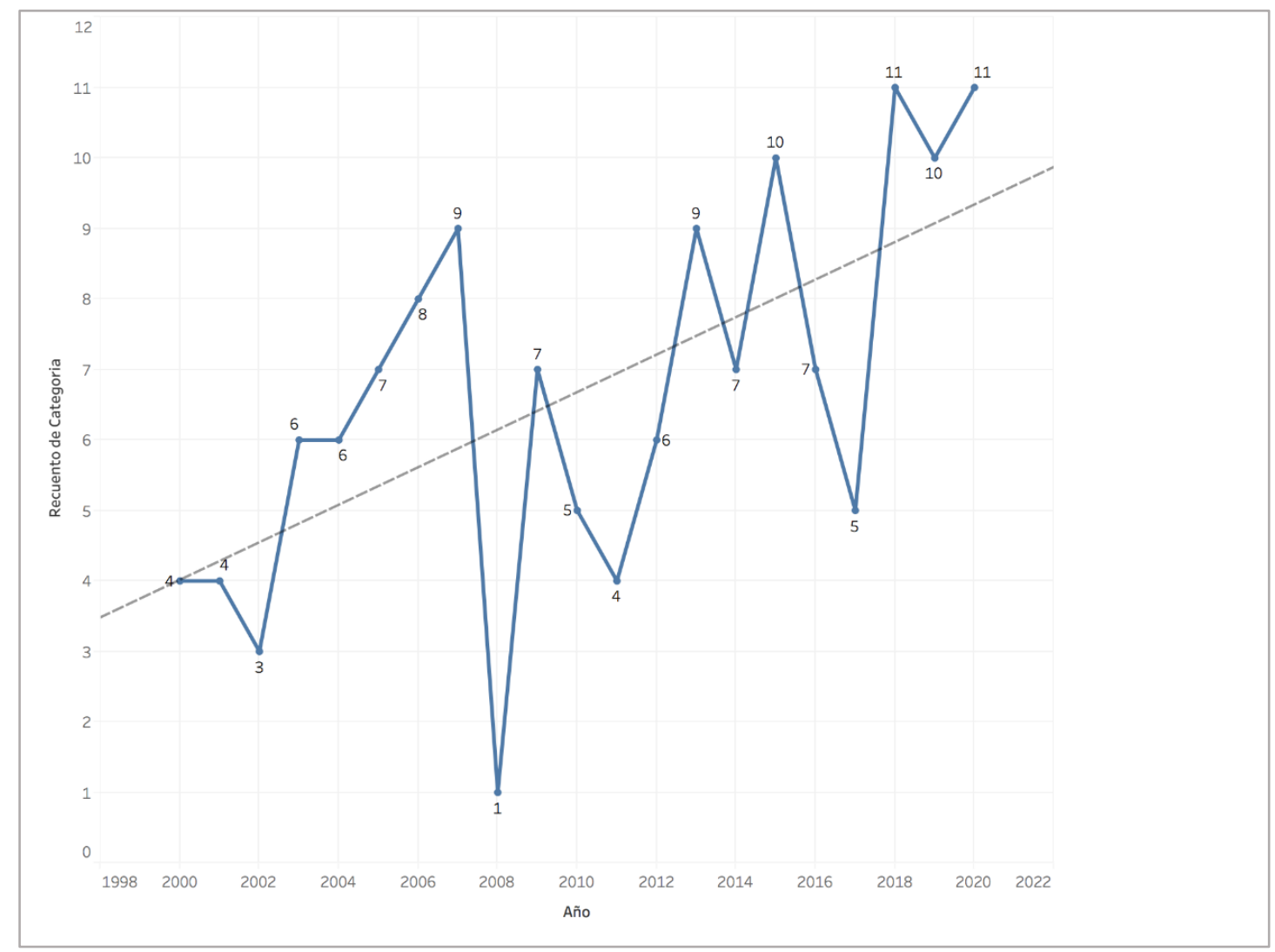

Fuente: Academy Awards Data Base

Tal y como se puede apreciar en la gráfica, desde el año en el que se inicia el presente análisis hasta el presente, la concesión de premios a mujeres en diversas categorías

RAE-IC, Revista de la Asociación Española de Investigación de la Comunicación vol. 8, núm. 16 (2021), 51-71 
tiene una tendencia alcista en la que se contabilizan algunas fluctuaciones con caídas significativas. Una vez efectuado el tratamiento de los datos para discriminar las categorías en las que existe diferenciación por género (Mejor Actriz Protagonista y Mejor Actriz en papel secundario) la línea de tendencia obtenidos ilustra un incremento desde los cuatro galardones en el año 2000 hasta los once premios contabilizados en el año 2020.

También se observan descensos importantes como el del año 2008 con solo una mujer premiada. Este dato resulta de interés ya que sucede justo después de uno de los máximos históricos, como son las nueve estatuillas entregadas a mujeres en el año 2007. En el año 2009, nuevamente se experimentó un ascenso hasta los siete premios y es, además, el año en el que Kathryn Bigelow recibe el Oscar a la Mejor Dirección, siendo la primera vez que una mujer recibe un premio en esta categoría.

En el año 2017 solo cinco mujeres recibieron un Oscar. Este año se inicia el movimiento \#MeToo que protagoniza la edición del certamen en el año 2018. Sin embargo, continúa la infrarrepresentación femenina y, aunque es una de las cifras más altas de la historia de los premios, solo trece mujeres se suben al estrado como ganadoras, once al descontar las categorías destinadas a las actrices.

La cifra más elevadas son los años 2018 y 2020 con once galardones y el año 2019 con diez. Dado el número de categorías y la trayectoria de los premios se confirma la dificultad que supone para la mujer el acceso a una representatividad paritaria en uno de los eventos más importantes de la industria cinematográfica, en el que solo a partir del activismo suscitado tras el movimiento social \#MeToo se han ido incrementando las cifras de premiadas pero sin llegar de momento a la paridad. 
Tabla 2. Categorías en la que han resultado premiadas más mujeres durante el período 2000-2020. Elaboración propia.

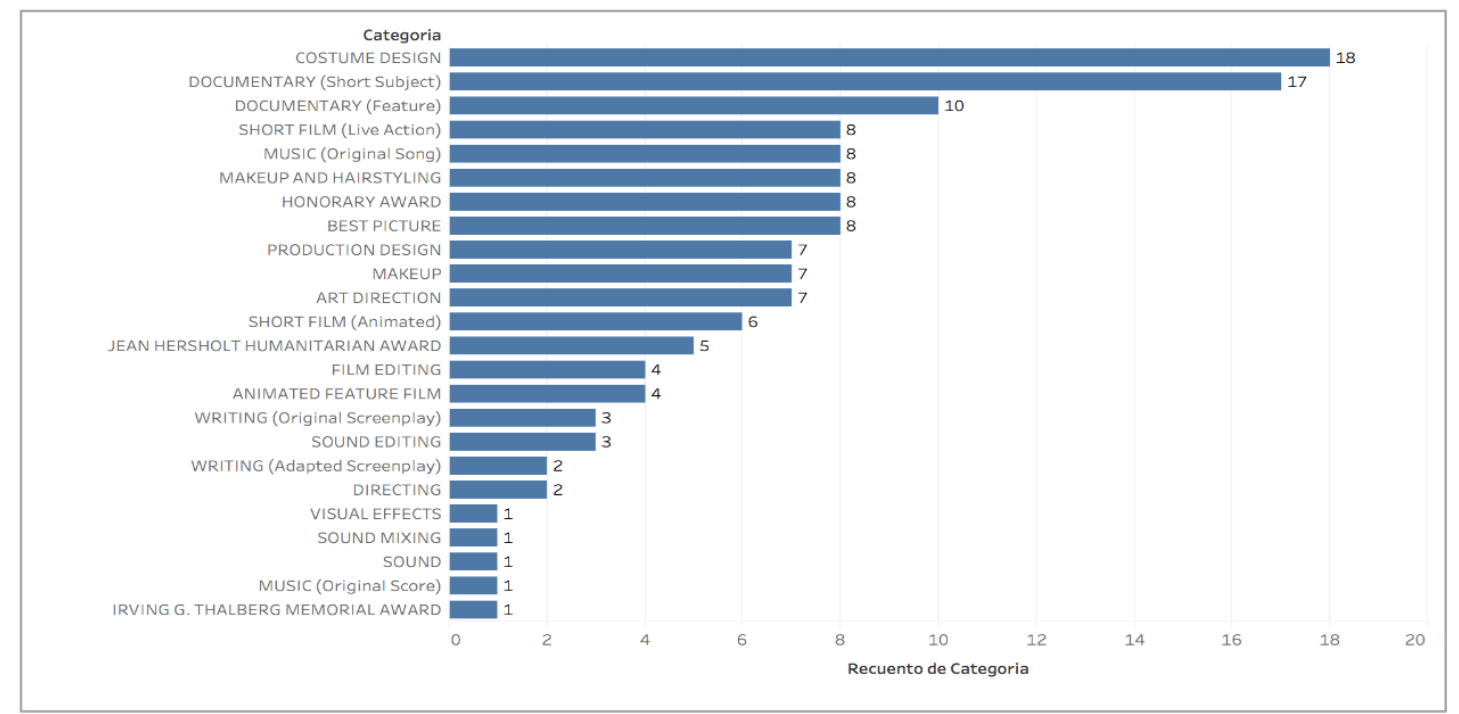

Fuente: Academy Awards Data Base.

Cuando examinamos los datos distribuidos en categorías se observa un sesgo de género en las categorías consideradas principales o, al menos, las más populares están reservadas a los hombres. La Tabla 2 representa los premios concedidos a mujeres, pero sin discriminar aquellos que son compartidos por varios profesionales.

En los veinte años que comprende el segmento temporal analizado, solo dos mujeres han recibido el Oscar a la Mejor Dirección y solo dos han sido las premiadas con el Mejor Guion Adaptado y tres con el Mejor Guion Original. Aunque suele ser habitual que la Mejor Película y la Mejor Dirección coincidan, los datos analizados muestran que mientras que la mejor Película se ha premiado en ocho ocasiones a una mujer, solo dos han sido premiadas como directoras, lo que significa que en seis ocasiones el Oscar a la Mejor Película fue para una mujer mientras que la Mejor Dirección fue otorgada a un hombre.

Las categorías en las que han resultado más premiadas las mujeres son la de Mejor Diseño de Vestuario (18 premios), Mejor Cortometraje Documental y Mejor Largometraje Documental (17 y 10 premios respectivamente). También es destacable el número de mujeres que han sido premiadas en categorías como Mejor Cortometraje o Mejor Maquillaje y Peluquería. Estos números ponen de manifiesto que las mujeres destacan en las categorías consideradas menores o en aquellas que se corresponden 
con profesiones muy feminizadas dentro del sector, tales como Maquillaje, Peluquería y Vestuario (Roquero, 2012).

Tabla 3. Categorías en las que se premian únicamente mujeres en premios no compartidos con hombres. Elaboración propia.

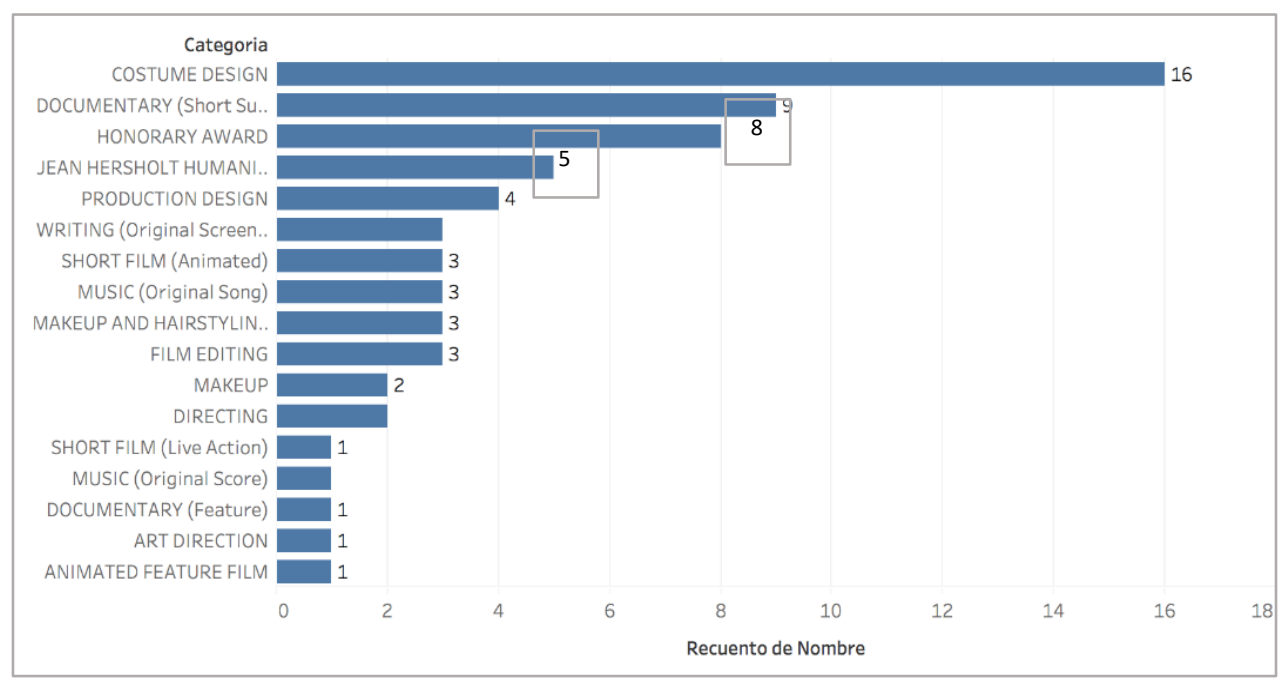

Fuente: Academy Awards Data Base

La Tabla 3 analiza la diferencia entre los premios concedidos solo a mujeres a lo largo de veinte años que representan un total de 66 galardones de un total de 182 en los que se premian mujeres, pero son compartidos. Al comparar los gráficos Tabla 2 y Tabla 3 , podemos observar cómo existe un gran número de premios compartidos en los que intervienen hombres limitando la visibilidad del reconocimiento a las mujeres en la industria audiovisual.

Las películas que aglutinan más premios Oscar concedidos a mujeres y a equipos que integran a mujeres son Mad Max: Fury Road (Miller, 2015), con cuatro galardones, The Hurt Locker (Bigelow, 2008) y The Aviator (Scorsesse, 2004) con tres estatuillas concedidas. Estas películas tienen un rango temático de lo que de forma habitual se relaciona con el espectro masculino, cine de acción, escenas violentas y mucho dinamismo (Yates, 2017). O también, como en el caso de The Aviator (Scorsesse, 2004) la propuesta se desarrolla con un protagonista masculino. Es posible que esta información se pueda apreciar con cierta subjetividad ya que al tratarse de un biopic sobre el magnate Howard Hughes, no existe una equivalencia en la que se pueda proponer una protagonista femenina, pero sí es posible analizar los personajes 
secundarios femeninos en los que se vuelve a reproducir la dialéctica en la que la mujer responde a roles y arquetipos tan habituales como la femme fatale, mujeres pasivas o mujeres con un carácter dominante que han sido masculinizadas en sus actitudes o atuendos (Checa y Adell, 2019).

\section{CONCLUSIONES}

El estudio de la representación y la representatividad de la mujer en la industria cinematográfica resulta de gran interés para entender cómo ha evolucionado el rol de las mujeres como profesionales y como personajes a lo largo de la historia del cine.

Resulta obvio el hecho de que tradicionalmente ha existido una proyección de lo femenino como sujetos pasivos o cosificados cuyos roles se articulaban como acompañantes del protagonista masculino (Checa y Adell, 2019). Al aplicar metodologías de análisis como el Test de Bechdel (Kelley, 2014) o más exhaustivas como el procesamiento mediante machine learning de los contenidos de las películas (Zhao, 2019), se observa como el desarrollo de los personajes masculinos y femeninos aún se sustenta sobre la desigualdad y tiene a reproducir arquetipos simplificados propios de una concepción patriarcal del papel de la mujer.

Tal y como plantea Guarinos (2008) existe una tendencia a creer que el relato audiovisual es más igualitario cuando los personajes femeninos desempeñan roles masculinos o tienen actitudes masculinas. Esta tendencia parece replicarse a la hora de otorgar reconocimiento a la mujer profesional ya que cuando han resultado premiadas mujeres suelen ser contenidos que se asocian tradicionalmente a gustos masculinos como, por ejemplo, el caso del Oscar a las Mejor Película y a la Mejor Dirección a Kathryn Bigelow por una película bélica en la que no abundan personajes femeninos protagonistas.

La representación de la mujer delante de la cámara proviene de la forma en que la sociedad concibe los roles asociados a lo femenino. Estas proyecciones arquetípicas tienen su origen en el relato patriarcal, pero cuando se plantea la pregunta de cómo sería posible la transformación de estas visiones de lo masculino y lo femenino en un 
plano más igualitarios, se observa que detrás de las cámaras la toma de decisiones recae en el género masculino en unas cifras abrumadoras.

Este hecho conduce al planteamiento de que difícilmente podrá existir un desarrollo más feminista del cine si la representatividad de la mujer en la profesión audiovisual se encuentra en inferioridad de condiciones. Para tratar de estimar la dificultad del acceso que experimentan las mujeres en el desarrollo de todas las facetas de los proyectos cinematográficos, esta investigación ha propuesto establecer un punto de inicio en el análisis del reconocimiento que la industria otorga al talento femenino. Partiendo de esta premisa, los objetivos de investigación se han centrado en analizar la frecuencia, en qué categorías y de qué manera son las mujeres premiadas en los festivales de cine tomando como ejemplo los últimos veinte años de los premios de la Academia de las Artes y Ciencias Cinematográficas, los Oscar.

De forma habitual se habla del techo de cristal para ilustrar las dificultades que experimentan las mujeres en el acceso al reparto de puestos de responsabilidad o de toma de decisiones en un sector determinado (Alonso-González, 2021). Otra fórmula que da testimonio de la posición que ostenta el género femenino en un determinado sector profesional es a través del reconocimiento que recibe por el ejercicio de una profesión determinada. Este suscita un debate sobre la relación o causalidad entre la infrarrepresentación y el talento. Sin embargo, al estudiar en profundidad los premios concedidos a mujeres en los Oscar de Hollywood, es posible inferir como la ausencia de mujeres en las categorías principales o las que son más populares entre el público y los medios de comunicación, se debe a que se trata de un entorno profundamente masculinizado, a pesar de que nos encontramos en medio de una época de transformación al abrigo de la cuarta ola feminista.

Con todo, las mujeres obtienen mayor reconocimiento en categorías consideradas menores y relacionadas con profesiones feminizadas como el diseño de vestuario, o en proyectos de menor envergadura como el documental o el cortometraje, no en el sentido de importancia sino en la utilización de recursos, distribución y marketing. 
Teniendo en cuenta estas consideraciones, los resultados del análisis efectuado vienen a confirmar la existencia de un sesgo de género en la concesión de premios a las mujeres cineastas. Esta ausencia de mujeres en todas las categorías se explica por los bajos índices de mujeres en determinadas profesiones dando lugar a un círculo vicioso en el que no se premia porque no abundan mujeres en determinados círculos y no abundan mujeres porque en el ascenso a las posiciones de poder interviene el techo de cristal. El resultado se traduce en que no hay una cuota paritaria en la industria audiovisual. Tal y como demuestran los datos analizados es complicado encontrar mujeres premiadas en la Dirección, en Guion, Efectos especiales o Banda Sonora o Dirección de fotografía o Diseño de Producción, y mucho más complicado aún encontrar mujeres premiadas de forma individual en dichas categorías, ya que si es posible hallar equipos mixtos.

De una u otra forma, la entrega de premios más famosa del cine a nivel mundial apenas ha visibilizado el talento femenino y esto conlleva que la percepción social sea que hacer cine es una cuestión en la que predomina el género masculino. Este debate conduce a un terreno farragoso en el que se cuestiona si el reconocimiento en forma de galardones de la magnitud de los Oscar es casual o causal de la representación de las mujeres detrás de la cámara. Los números parecen indicar que la escasa participación femenina está relacionada con el acceso al ejercicio de la profesión y que para que esta cuestión pueda cambiar es preciso que las mujeres tengan acceso al desarrollo de todas las facetas que intervienen en la realización de una película.

Mujeres escribiendo, dirigiendo, produciendo, montando, editando y, en definitiva, mujeres trabajando en todos los sectores de la industria se traducirá en una transformación del discurso narrativo audiovisual, en una evolución de los arquetipos y en más mujeres recogiendo premios debido a una cuestión de igualdad en el acceso y de representación paritaria.

Dadas las limitaciones del estudio, cabe destacar que sería de gran interés para futuras investigaciones evaluar estas mismas tendencias en diferentes festivales, premios y eventos para observar si la ausencia del principio de universalidad se produce y de qué forma en los diferentes mercados cinematográficos. 


\section{RECONOCIMIENTO}

Los resultados de este artículo forman parte de las actividades de investigación promovidas a través del proyecto de investigación RTI2018-096065-B-I00 del Programa Estatal de $|+D+|$ orientado a los Retos de la Sociedad del Ministerio de Ciencia, Innovación y Universidades y del Fondo Europeo de Desarrollo Regional (FEDER) sobre "Nuevos valores, gobernanza, financiación y servicios audiovisuales públicos para la sociedad de Internet: contrastes europeos y españoles".

\section{REFERENCIAS BIBLIOGRÁFICAS}

Academy of Motion Picture Arts and Sciencie (2021). Academy Awards Search Data Base. Recuperado de http://awardsdatabase.oscars.org

Alonso-González, M. (2021). Activismo social femenino en la esfera pública digital. Vivat Academia, 154, 133-156. https://doi.org/10.15178/va.2021.154.e1239

Álvarez-Hernández, C., González de Garay-Domínguez, B. y Frutos-Esteban, F.J. (2015). Representación de género. Las películas españolas contemporáneas de adolescentes (2009-2014). Revista Latina de Comunicación Social, 70, 934-960. Recuperado de http://www.revistalatinacs.org/070/paper/1079/49es.html

Annerberg Foundation (2020). Inequality in 1,300 Popular Films: Examining Portrayals of Gender, Race/Ethnicity, LGBTQ \& Disability from 2007 to 2019. Annerberg Inclusion Initiative.

Bazo Varela, E. (2003). Las claves audiovisuales del 11 de septiembre. Revista Latente, 1, 97-105.

Berrueta, J. (20 de noviembre de 2019). Las mujeres ocultas de la historia del cine: el brutal documental que enseña el machismo de la industria. El Español. Recuperado de https://www.elespanol.com/cultura/cine/20191120/mujeres-ocultas-historia-brutaldocumental-machismo-industria/445706428_0.html 
Cabrera Blázquez, J., Cappello, M., Talavera Milla, J. y Valais, S. (2021). Diversity and Inclusion in the European Audiovisual Sector. Iris Plus. Strasbourg: European Audiovisual Observatory.

Castejón Leorza, M. (2004). Mujeres y Cine. Las fuentes cinematográficas para el avance de la historia de las mujeres. Berceo, 147, 303-327.

Checa Bañuz, C. y Adell Carmona, M. (2019). "Perfectas para el papel”: representaciones de las mujeres científicas en la ciencia-ficción contemporánea. En WSCITECH2019, "Congrés Dones Ciència i Tecnologia 2019. Terrassa, 6 i 7 de marzo de 2019".

Cousins, M. (2018). Women Make Film: A New Road Movie Through Cinema [serie documental]. Reino Unido: Dogwoof Digital, Hopscotch Films

Cuenca Suárez, S. (2019). Informe Anual CIMA. La representatividad de las mujeres en el sector cinematográfico del largometraje español: 2019.

El País (5 de enero de 2020). Revolución \#Metoo, cronología de un movimiento global. El País. Recuperado de https://elpais.com/especiales/movimiento-metoo/cronologia/

Fontaine, G. y Simone, P. (2020). Female directors and screenwritters in European film and audiovisual fiction production. European Audiovisual Observatory. Recuperado de www.obs.coe.int

Gaitán Moya, J. A. y Piñuel Raigada, J. L. (1998). Técnicas de investigación en comunicación social. Madrid: Síntesis.

Gómez-Diago, G. (2010). Triangulación metodológica: paradigma para investigar desde la ciencia de la comunicación. Razón y Palabra, 72.

Guarinos, V. (2008). Mujer y Cine. En T. Nuñez et al. (Eds.), Los Medios de Comunicación con Mirada de Género (pp. 103-120). Sevilla. Instituto Andaluz de la Mujer.

Hernández-Carrillo, C. y Ruiz del Olmo, F. J. (2018). Presencia y visibilidad de la mujer en la producción cinematográfica española: estudio de los filmes galardonados en el 
festival de Málaga de cine en español. En A. I. Arévalo Salinas, G. Vilar Sastre y T. Al Najjar Trujillo (Eds.), Comunicación, paz y conflictos (pp. 195-203). Madrid: Dykinson.

Okuda Benavides, M. y Gómez-Restrepo, C. (2005). Métodos de investigación cualitativa: triangulación. Revista Colombiana de Psiquiatría, 34(1).

Kelley, M. J. (2014). Mirrored discourse in Alison Bechdel Fun Home. Journal of Graphic Novels and Comics, 5(1), 42-57. https://doi.org/10.1080/21504857.2013.826263

Lauzen, Martha M. (2019). The celluloid ceiling: Behind-the-Scenes Employment of Women on the Top 100, 250, and 500 Films of 2018. Recuperado de https://cutt.ly/IE5Z6VK

Roquero, E. (2012). El «techo de cristal» en la dirección cinematográfica: discursos y representaciones sociales en un caso de segregación ocupacional. Mediaciones Sociales 10, 37-61. http://dx.doi.org/10.5209/rev_MESO.2012.n10.39681

Saavedra, J. M. (2019). Una nueva ola feminista... más allá de \#MeToo. Irrupción, legado y desafíos. Políticas Públicas para la Equidad, 2.

WSCITECH (2019). Libre de actes Congrés Dones, Ciència i Tecnol.gía, Terrassa 6 y 7 de marzo. https://doi.org/10.3926/wscitech19

Yates, M. (2017). Re-casting nature as a feminist space in Mad Max: Fury Road. Science Fiction Film and Television, 10(3), 353-70

Zhao, T. (2019). Mind the Gap: Understanding Gender Inequality in Movie Industry using Social Network Analysis and Machine Learning (pp. 100-106). High School Attached to Northwest Normal University, Gansu, China: Institute of Electrical and Electronics Engineers Inc. https://doi.org/10.1109/ITCA49981.2019.00030 